\title{
Coefficient Estimates of Bi-Starlike and Bi-Convex Functions with Respect to Symmetrical Points Associated with the Second Kind Chebyshev Polynomials
}

\author{
Abbas Kareem Wanas \\ Department of Mathematics, College of Science, University of Al-Qadisiyah, Diwaniyah, Iraq \\ e-mail: abbas.kareem.w@qu.edu.iq
}

\begin{abstract}
In this paper, by making use the second kind Chebyshev polynomials, we introduce and study a certain class of bi-starlike and bi-convex functions with respect to symmetrical points defined in the open unit disk. We find upper bounds for the second and third coefficients of functions belong to this class.
\end{abstract}

\section{Introduction}

The importance of Chebyshev polynomial in numerical analysis is increased in both theoretical and practical points of view. There are four kinds of Chebyshev polynomials. Several researchers dealing with orthogonal polynomials of Chebyshev family, contain mainly results of Chebyshev polynomials of first kind $T_{n}(t)$, the second kind $U_{n}(t)$ and their numerous uses in different applications one can refer $[5,7,9]$. The Chebyshev polynomials of the first and second kinds are well known and they are defined by

$$
T_{n}(t)=\cos n \theta \quad \text { and } \quad U_{n}(t)=\frac{\sin (n+1) \theta}{\sin \theta} \quad(-1<t<1),
$$

where $n$ indicates the polynomial degree and $t=\cos n \theta$.

Received: November 27, 2019; Accepted: February 11, 2020

2010 Mathematics Subject Classification: 30C45, 30C50.

Keywords and phrases: bi-univalent function, coefficient estimates, Chebyshev polynomials, subordination.

Copyright (C) 2020 Abbas Kareem Wanas. This is an open access article distributed under the Creative Commons Attribution License, which permits unrestricted use, distribution, and reproduction in any medium, provided the original work is properly cited. 
Let $\mathcal{A}$ stand for the family of functions $f$ which are analytic in the open unit disk $U=\{z \in \mathbb{C}:|z|<1\}$ that have the form:

$$
f(z)=z+\sum_{n=2}^{\infty} a_{n} z^{n} .
$$

Also, let $S$ be the subclass of $\mathcal{A}$ consisting of the form (1.1) which are univalent in $U$. It is well known (see [6]) that every function $f \in S$ has an inverse $f^{-1}$, defined by

$$
\begin{gathered}
f^{-1}(f(z))=z,(z \in U) \text { and } f\left(f^{-1}(w)\right)=w,\left(|w|<r_{0}(f), r_{0}(f) \geq \frac{1}{4}\right), \text { where } \\
\quad g(w)=f^{-1}(w)=w-a_{2} w^{2}+\left(2 a_{2}^{2}-a_{3}\right) w^{3}-\left(5 a_{2}^{3}-5 a_{2} a_{3}+a_{4}\right) w^{4}+\ldots .
\end{gathered}
$$

A function $f \in \mathcal{A}$ is said to be bi-univalent in $U$ if both $f$ and $f^{-1}$ are univalent in $U$. Let $\sum$ stand for the class of bi-univalent functions in $U$ given by (1.1). For a brief history and interesting examples of functions that are in (or are not in) the class $\Sigma$, together with various other properties of the bi-univalent functions class $\Sigma$, one can refer the work of Srivastava et al. [13] and the references stated therein. Recently, many authors introduced various subclasses of the bi-univalent functions class $\sum$ and investigated non sharp estimates on the first two coefficients $\left|a_{2}\right|$ and $\left|a_{3}\right|$ in the Taylor-Maclaurin series expansion (1.1) (see [1, 2, 3, 4, 8, 12]).

Sakaguchi [11] introduced the class $S_{s}^{*}$ of functions starlike with respect to symmetric points, which consists of functions $f \in S$ satisfying the condition

$$
\operatorname{Re}\left\{\frac{z f^{\prime}(z)}{f(z)-f(-z)}\right\}>0, \quad z \in U .
$$

Also, Wang et al. [15] introduced the class $K_{s}$ of functions convex with respect to symmetric points, which consists of functions $f \in S$ satisfying the condition

$$
\operatorname{Re}\left\{\frac{\left(z f^{\prime}(z)\right)^{\prime}}{(f(z)-f(-z))^{\prime}}\right\}>0, \quad z \in U .
$$

With a view to recalling the principal of subordination between analytic functions, let the functions $f$ and $g$ be analytic in $U$. We say that the function $f$ is said to be 
subordinate to $g$, if there exists a Schwarz function $w$ analytic in $U$ with $w(0)=0$ and $|w(z)|<1(z \in U)$ such that $f(z)=g(w(z))$. This subordination is denoted by $f \prec g$ or $f(z) \prec g(z)(z \in U)$. It is well known that (see [10]), if the function $g$ is univalent in $U$, then $f \prec g$ if and only if $f(0)=g(0)$ and $f(U) \subset g(U)$.

We consider the function

$$
H(z, t)=\frac{1}{1-2 t z+z^{2}}, t \in\left(\frac{1}{2}, 1\right], z \in U
$$

We note that if $t=\cos \beta$, where $\beta \in\left(-\frac{\pi}{3}, \frac{\pi}{3}\right)$, then

$$
H(z, t)=\frac{1}{1-2 \cos \beta z+z^{2}}=1+\sum_{n=1}^{\infty} \frac{\sin (n+1) \beta}{\sin \beta} z^{n}, \quad z \in U
$$

Therefore

$$
H(z, t)=1+2 \cos \beta z+\left(3 \cos ^{2} \beta-\sin ^{2} \beta\right) z^{2}+\cdots, \quad z \in U .
$$

In view of [16], we can write

$$
H(z, t)=1+U_{1}(t) z+U_{2}(t) z^{2}+\cdots \quad(z \in U, t \in(-1,1)),
$$

where

$$
U_{n-1}=\frac{\sin (n \arccos t)}{\sqrt{1-t^{2}}} \quad(n \in \mathbb{N}=\{1,2, \ldots\})
$$

are the Chebyshev polynomials of the second kind. Also, it is known that

$$
U_{n}(t)=2 t U_{n-1}(t)-U_{n-2}(t)
$$

and

$$
U_{1}(t)=2 t, \quad U_{2}(t)=4 t^{2}-1, \quad U_{3}(t)=8 t^{3}-4 t, \ldots .
$$

The generating function of the first kind of Chebyshev polynomial $T_{n}(t), t \in[-1,1]$ is given by

$$
\sum_{n=0}^{\infty} T_{n}(t) z^{n}=\frac{1-t z}{1-2 t z+z^{2}}, \quad z \in U
$$


The Chebyshev polynomials of first kind $T_{n}(t)$ and of the second kind $U_{n}(t)$ are connected by

$$
\frac{d T_{n}(t)}{d t}=n U_{n-1}(t), T_{n}(t)=U_{n}(t)-t U_{n-1}(t), 2 T_{n}(t)=U_{n}(t)-U_{n-2}(t) .
$$

\section{Main Results}

Definition 2.1. For $\gamma \geq 0$ and $t \in\left(\frac{1}{2}, 1\right]$, a function $f \in \Sigma$ is said to be in the class $\mathcal{D}_{\Sigma}^{s}(\gamma, t)$ if it satisfies the subordinations:

$$
\left(\frac{2 z f^{\prime}(z)}{f(z)-f(-z)}\right)^{\gamma}\left(\frac{2\left(z f^{\prime}(z)\right)^{\prime}}{(f(z)-f(-z))^{\prime}}\right)^{1-\gamma} \prec H(z, t)=\frac{1}{1-2 t z+z^{2}}
$$

and

$$
\left(\frac{2 w g^{\prime}(w)}{g(w)-g(-w)}\right)^{\gamma}\left(\frac{2\left(w g^{\prime}(w)\right)^{\prime}}{(g(w)-g(-w))^{\prime}}\right)^{1-\gamma} \prec H(w, t)=\frac{1}{1-2 t w+w^{2}}
$$

where the function $g=f^{-1}$ is given by (1.2).

Theorem 2.1. For $\gamma \geq 0$ and $t \in\left(\frac{1}{2}, 1\right]$, let f be in the class $\mathcal{D}_{\Sigma}^{s}(\gamma, t)$. Then

$$
\left|a_{2}\right| \leq \frac{t \sqrt{2 t}}{\sqrt{\left|2(\gamma-1) t^{2}-(\gamma-2)^{2}\left(2 t^{2}-1\right)\right|}}
$$

and

$$
\left|a_{3}\right| \leq \frac{t^{2}}{(\gamma-2)^{2}}+\frac{t}{3-2 \gamma}
$$

Proof. Let $f \in \mathcal{D}_{\Sigma}^{s}(\gamma, t)$. Then there exists two analytic functions $u, v: U \rightarrow U$ given by

$$
u(z)=u_{1} z+u_{2} z^{2}+u_{3} z^{3}+\cdots \quad(z \in U)
$$


and

$$
v(w)=v_{1} w+v_{2} w^{2}+v_{3} w^{3}+\cdots \quad(w \in U),
$$

with $u(0)=v(0)=0,|u(z)|<1,|v(w)|<1, w \in U$ such that

$$
\left(\frac{2 z f^{\prime}(z)}{f(z)-f(-z)}\right)^{\gamma}\left(\frac{2\left(z f^{\prime}(z)\right)^{\prime}}{(f(z)-f(-z))^{\prime}}\right)^{1-\gamma}=1+U_{1}(t) u(z)+U_{2}(t) u^{2}(z)+\cdots
$$

and

$$
\left(\frac{2 w g^{\prime}(w)}{g(w)-g(-w)}\right)^{\gamma}\left(\frac{2\left(w g^{\prime}(w)\right)^{\prime}}{(g(w)-g(-w))^{\prime}}\right)^{1-\gamma}=1+U_{1}(t) v(w)+U_{2}(t) v^{2}(w)+\cdots .
$$

Combining (2.1), (2.2), (2.3) and (2.4), we obtain

$$
\begin{aligned}
& \left(\frac{2 z f^{\prime}(z)}{f(z)-f(-z)}\right)^{\gamma}\left(\frac{2\left(z f^{\prime}(z)\right)^{\prime}}{(f(z)-f(-z))^{\prime}}\right)^{1-\gamma} \\
= & 1+U_{1}(t) u_{1} z+\left[U_{1}(t) u_{2}+U_{2}(t) u_{1}^{2}\right] z^{2}+\cdots
\end{aligned}
$$

and

$$
\begin{aligned}
& \left(\frac{2 w g^{\prime}(w)}{g(w)-g(-w)}\right)^{\gamma}\left(\frac{2\left(w g^{\prime}(w)\right)^{\prime}}{(g(w)-g(-w))^{\prime}}\right)^{1-\gamma} \\
= & 1+U_{1}(t) v_{1} w+\left[U_{1}(t) v_{2}+U_{2}(t) v_{1}^{2}\right] w^{2}+\cdots
\end{aligned}
$$

It is well-known that if $|u(z)|<1$ and $|v(w)|<1, z, w \in U$, then

$$
\left|u_{i}\right| \leq 1 \text { and }\left|v_{i}\right| \leq 1 \text { for all } i \in \mathbb{N} .
$$

Comparing the corresponding coefficients in (2.5) and (2.6), after simplifying, we have

$$
\begin{gathered}
-2(\gamma-2) a_{2}=U_{1}(t) u_{1}, \\
2\left[(\gamma-2)^{2}+(3 \gamma-4)\right] a_{2}^{2}+2(3-2 \gamma) a_{3}=U_{1}(t) u_{2}+U_{2}(t) u_{1}^{2}, \\
2(\gamma-2) a_{2}=U_{1}(t) v_{1}
\end{gathered}
$$


and

$$
2\left[(\gamma-2)^{2}+(5-3 \gamma)+(2 \gamma-3)\right] a_{2}^{2}+2(2 \gamma-3) a_{3}=U_{1}(t) v_{2}+U_{2}(t) v_{1}^{2} .
$$

It follows from (2.8) and (2.10) that

$$
u_{1}=-v_{1}
$$

and

$$
8(\gamma-2)^{2} a_{2}^{2}=U_{1}^{2}(t)\left(u_{1}^{2}+v_{1}^{2}\right)
$$

If we add (2.9) to (2.11), we find that

$$
2\left(2(\gamma-2)^{2}+2(\gamma-1)\right) a_{2}^{2}=U_{1}(t)\left(u_{2}+v_{2}\right)+U_{2}(t)\left(u_{1}^{2}+v_{1}^{2}\right) .
$$

Substituting the value of $u_{1}^{2}+v_{1}^{2}$ from (2.13) in the right hand side of (2.14), we get

$$
4\left[(\gamma-2)^{2}\left(1-\frac{2 U_{2}(t)}{U_{1}^{2}(t)}\right)+\gamma-1\right] a_{2}^{2}=U_{1}(t)\left(u_{2}+v_{2}\right) .
$$

Further computations using (1.3), (2.7) and (2.15), we obtain

$$
\left|a_{2}\right| \leq \frac{t \sqrt{2 t}}{\sqrt{\left|2(\gamma-1) t^{2}-(\gamma-2)^{2}\left(2 t^{2}-1\right)\right|}} .
$$

Next, if we subtract (2.11) from (2.9), we deduce that

$$
4(3-2 \gamma)\left(a_{3}-a_{2}^{2}\right)=U_{1}(t)\left(u_{2}-v_{2}\right)+U_{2}(t)\left(u_{1}^{2}-v_{1}^{2}\right) .
$$

In view of (2.12) and (2.13), we get from (2.16)

$$
a_{3}=\frac{U_{1}^{2}(t)}{8(\gamma-2)^{2}}\left(u_{1}^{2}+v_{1}^{2}\right)+\frac{U_{1}(t)}{4(3-2 \gamma)}\left(u_{2}-v_{2}\right) .
$$

Thus applying (1.3), we obtain

$$
\left|a_{3}\right| \leq \frac{t^{2}}{(\gamma-2)^{2}}+\frac{t}{3-2 \gamma} .
$$

For $\gamma=1$, the class $\mathcal{D}_{\Sigma}^{s}(\gamma, t)$ reduced to the class $\mathcal{D}_{\Sigma}^{s}(1, t)$ of bi-starlike functions 
with respect to symmetrical points. For functions belongs to this class, we conclude the following result.

Corollary 2.1. For $t \in\left(\frac{1}{2}, 1\right]$, let f be in the class $\mathcal{D}_{\Sigma}^{s}(1, t)$. Then

$$
\left|a_{2}\right| \leq \frac{t \sqrt{2 t}}{\sqrt{\left|2 t^{2}-1\right|}}
$$

and

$$
\left|a_{3}\right| \leq t(t+1)
$$

For $\gamma=0$, the class $\mathcal{D}_{\Sigma}^{s}(\gamma, t)$ reduced to the class $\mathcal{F}_{\Sigma}^{s c}(t)$ which was considered recently by Wanas and Majeed [14].

Corollary 2.2 [14]. For $t \in\left(\frac{1}{2}, 1\right]$, let f be in the class $\mathcal{F}_{\Sigma}^{s c}(t)$. Then

$$
\left|a_{2}\right| \leq \frac{t \sqrt{2 t}}{\sqrt{\left|2-5 t^{2}\right|}}
$$

and

$$
\left|a_{3}\right| \leq \frac{t(3 t+4)}{12}
$$

\section{References}

[1] R. M. Ali, S. K. Lee, V. Ravichandran and S. Supramaniam, Coefficient estimates for biunivalent Ma-Minda starlike and convex functions, Appl. Math. Lett. 25 (2012), 344-351. https://doi.org/10.1016/j.aml.2011.09.012

[2] Ş. Altınkaya and S. Yalçın, Coefficient estimates for two new subclasses of biunivalent functions with respect to symmetric points, J. Funct. Spaces 2015, Art. ID 145242, 5 pp. https://doi.org/10.1155/2015/145242

[3] Ş. Altınkaya and S. Yalçın, On a new subclass of bi-univalent functions of Sakaguchi type satisfying subordinate conditions, Malaya Journal of Mathematics 5(2) (2017), 305-309.

[4] M. Çağlar, H. Orhan and N. Yağmur, Coefficient bounds for new subclasses of biunivalent functions, Filomat 27 (2013), 1165-1171.

https://doi.org/10.2298/FIL1307165C 
[5] E. H. Doha, The first and second kind Chebyshev coefficients of the moments for the general order derivative of an infinitely differentiable function, Int. J. Comput. Math. 51 (1994), 21-35. https://doi.org/10.1080/00207169408804263

[6] P. L. Duren, Univalent Functions, Grundlehren der Mathematischen Wissenschaften, Band 259, Springer Verlag, New York, Berlin, Heidelberg and Tokyo, 1983.

[7] J. Dziok, R. K. Raina and J. Sokół, Application of Chebyshev polynomials to classes of analytic functions, C. R. Math. Acad. Sci. Paris 353 (2015), 433-438. https://doi.org/10.1016/j.crma.2015.02.001

[8] N. Magesh and V. Prameela, Coefficient estimate problems for certain subclasses of analytic and bi-univalent functions, Afr. Mat. 26(3) (2015), 465-470. https://doi.org/10.1007/s13370-013-0220-0

[9] J. C. Mason, Chebyshev polynomial approximations for the $L$-membrane eigenvalue problem, SIAM J. Appl. Math. 15 (1967), 172-186. https://doi.org/10.1137/0115014

[10] S. S. Miller and P. T. Mocanu, Differential Subordinations: Theory and Applications, Series on Monographs and Textbooks in Pure and Applied Mathematics, Vol. 225, Marcel Dekker Inc., New York and Basel, 2000.

[11] K. Sakaguchi, On a certain univalent mapping, J. Math. Soc. Japan 11(1) (1959), 72-75. https://doi.org/10.2969/jmsj/01110072

[12] H. M. Srivastava, S. Gaboury and F. Ghanim, Coefficient estimates for some general subclasses of analytic and bi-univalent functions, Afr. Math. 28 (2017), 693-706. https://doi.org/10.1007/s13370-016-0478-0

[13] H. M. Srivastava, A. K. Mishra and P. Gochhayat, Certain subclasses of analytic and biunivalent functions, Appl. Math. Lett. 23 (2010), 1188-1192. https://doi.org/10.1016/j.aml.2010.05.009

[14] A. K. Wanas and A. H. Majeed, Chebyshev polynomial bounded for analytic and biunivalent functions with respect to symmetric conjugate points, Appl. Math. E-Notes 19 (2019), 14-21.

[15] Z. G. Wang, C. Y. Gao and S. M. Yuan, On certain subclasses of close-to-convex and quasi-convex functions with respect to $k$-symmetric points, J. Math. Anal. Appl. 322(1) (2006), 97-106. https://doi.org/10.1016/j.jmaa.2005.08.060

[16] E. T. Whittaker and G. N. Watson, A Course of Modern Analysis, Cambridge: Cambridge Univ. Press, 1996. https://doi.org/10.1017/CBO9780511608759 